\title{
Clinical and parasitological response to oral chloroquine and primaquine in uncomplicated human Plasmodium knowlesi infections
}

Cyrus Daneshvar ${ }^{1}$, Timothy ME Davis², Janet Cox-Singh ${ }^{1}$, Mohammad Z Rafa'ee ${ }^{3}$, Siti K Zakaria', Paul CS Divis ${ }^{1}$, Balbir Singh ${ }^{*}$

\begin{abstract}
Background: Plasmodium knowlesi is a cause of symptomatic and potentially fatal infections in humans. There are no studies assessing the detailed parasitological response to treatment of knowlesi malaria infections in man and whether antimalarial resistance occurs.

Methods: A prospective observational study of oral chloroquine and primaquine therapy was conducted in consecutive patients admitted to Kapit Hospital, Sarawak, Malaysian Borneo with PCR-confirmed single P. knowlesi infections. These patients were given oral chloroquine for three days, and at 24 hours oral primaquine was administered for two consecutive days, primarily as a gametocidal agent. Clinical and parasitological responses were recorded at 6-hourly intervals during the first 24 hours, daily until discharge and then weekly to day 28. Vivax malaria patients were studied as a comparator group.

Results: Of 96 knowlesi malaria patients who met the study criteria, 73 were recruited to an assessment of the acute response to treatment and 60 completed follow-up over 28 days. On admission, the mean parasite stage distributions were $49.5 \%, 41.5 \%, 4.0 \%$ and $5.6 \%$ for early trophozoites, late trophozoites, schizonts and gametocytes respectively. The median fever clearance time was 26.5 [inter-quartile range 16-34] hours. The mean times to 50\% $\left(\mathrm{PCT}_{50}\right)$ and 90\% (PCT $\left.\mathrm{T}_{90}\right)$ parasite clearance were 3.1 (95\% confidence intervals [Cl] 2.8-3.4) hours and 10.3 (9.4-11.4) hours. These were more rapid than in a group of 23 patients with vivax malaria 6.3 (5.3-7.8) hours and 20.9 (17.625.9) hours; $P=0.02$ ). It was difficult to assess the effect of primaquine on $P$. knowlesi parasites, due to the rapid anti-malarial properties of chloroquine and since primaquine was administered 24 hours after chloroquine. No P. knowlesi recrudescences or re-infections were detected by PCR.
\end{abstract}

Conclusions: Chloroquine plus primaqine is an inexpensive and highly effective treatment for uncomplicated knowlesi malaria infections in humans and there is no evidence of drug resistance. Further studies using alternative anti-malarial drugs, including artemisinin derivatives, would be desirable to define optimal management strategies for P. knowlesi.

\section{Background}

The simian malaria parasite Plasmodium knowlesi causes symptomatic infections in humans throughout South-east Asia in areas inhabited by its natural macaque hosts (Macaca fascicularis and M. nemestrina) and mosquito vectors of the Anopheles leucosphyrus group

\footnotetext{
* Correspondence: bskhaira55@gmail.com

'Malaria Research Centre, Faculty of Medicine \& Health Sciences, Universiti

Malaysia Sarawak, 93150 Kuching, Sarawak, Malaysia

Full list of author information is available at the end of the article
}

[1-8]. Early trophozoites of P. knowlesi are morphologically similar to Plasmodium falciparum and all the other stages resemble those of Plasmodium malariae [9]. However, unlike P. malariae infections, knowlesi infections can be severe and even fatal. A recent observational study found complications in $10 \%$ of patients and a $2 \%$ mortality [10].

Previous reports have indicated that patients with uncomplicated knowlesi malaria respond well to chloroquine treatment [1]. In one recent study, the mean 
parasite clearance time of 24 hours for knowlesi malaria patients was shorter than that for patients infected with P. falciparum or Plasmodium vivax [9]. There has, however, been no detailed description of clinical and parasitological responses following treatment and no previous assessment of antimalarial drug resistance. Such a study was, therefore, undertaken on patients with knowlesi and vivax malaria during hospital stay and on a weekly basis for a month.

\section{Methods}

\section{Study site, design and patients}

The present study was conducted in Kapit Hospital, which serves a population of 109,000 in Sarawak, Malaysia Borneo, an area with a reported annual malaria incidence of 1.2/1,000. A prospective observational study design was employed, using the WHO 28 day extended in vivo drug response assessment [11]. The patients were selected from a larger cohort participating in a pilot study and a subsequent observational assessment of consecutive malaria admissions between May 2006 and December 2007 [10]. Although there are currently no criteria for severity of infection in knowlesi infected patients, we have previously defined uncomplicated cases as those with a parasitaemia of $\leq 100,000 / \mu \mathrm{L}$ based on falciparum malaria data and the level of immunity in the community. In addition for the present study, patients that were selected i) had P. knowlesi or P. vivax monoinfections confirmed by PCR, ii) were at least 15 years of age, iii) were not pregnant, and iv) had no significant co-morbidities. Those who reported treatment with antimalarial drugs within the preceding 14 days were excluded. All patients gave written informed consent to participation. The study was approved by the Medical Research and Ethics Committee of the Malaysian Ministry of Health.

\section{Antimalarial therapy}

All patients were given oral chloroquine $10 \mathrm{mg}$ base $/ \mathrm{kg}$ body weight, followed by $5 \mathrm{mg}$ base $/ \mathrm{kg}$ at 6,24 and 48 hours (total dose $25 \mathrm{mg}$ base $/ \mathrm{kg}$ ). At 24 hours, and after glucose-6-phosphate dehydrogenase deficiency had been excluded (SQMMR 720, R\&D diagnostics, Holargos Greece), oral primaquine (15 mg base) was administered with food for either two consecutive days for knowlesi malaria (primarily as a gametocidal agent, as recommended by the Malaysian Ministry of Health for patients with $P$. malariae; there were no treatment guidelines for $P$. knowlesi at the time of the study and PCR confirmation was pending) or 14 days for vivax malaria (as also recommended by the Malaysian Ministry of Health). All drugs were administered by nursing staff on the medical ward where the patient was observed until parasite clearance (when two consecutive blood films 24 hours apart were negative for malaria parasites).

\section{Clinical and laboratory procedures}

At recruitment, a detailed history and clinical examination were performed and recorded on standardized forms. Blood samples were taken for routine laboratory investigations including a full blood count, biochemical analyses, and blood cultures. In view of the possibility of cardio-respiratory complications developing during treatment [10], a baseline chest radiograph and 12 lead electrocardiogram were performed.

On admission and daily thereafter during hospitalisation, blood samples were collected on filter paper for subsequent DNA extraction and polymerase chain reaction (PCR) analyses to identify the species of Plasmodium and determine parasite clearance as described previously $[1,9,12]$. Blood was drawn for a full blood count at 0 and 24 hours. All patients had four-hourly monitoring of vital signs, daily clinical examination, daily blood sampling and blood films until parasite and fever clearance.

In randomly-selected sub-groups of patients with knowlesi or vivax malaria, additional samples were collected at three or six-hourly intervals for the first 24 hours to enable more detailed characterisation of initial parasite clearance. Because of the limited number of patients with $P$. vivax infections, twice the proportion was selected for this group compared to that in the P. knowlesi group.

Following discharge, patients were reviewed on days 7 , 14, 21 and 28. Symptoms and clinical signs including the presence of splenomegaly or hepatomegaly were recorded, and further blood samples were taken, including blood films and filter paper samples for PCR analyses. If returning to Kapit Hospital for weekly visits was difficult, patients were asked to attend their nearest clinic or prepare their own blood spots on filter papers on days 7, 14 and 21, and to return on day 28 for full review. When patients did not attend a follow up appointment, efforts were made to contact them via telephone, through their employer if at a logging camp, through people from the same region who were seen at Kapit Hospital, or by use of the local radio station. Patients were excluded from the study if they missed a follow-up visit.

All blood films were examined by two microscopists who were blinded to all clinical and other data. Parasite counts were calculated from the number of parasites per 500 white cells, and a density calculated using the patient's own white cell count. Stages were classified as early trophozoites (ring forms and non-pigmented trophozoites), late trophozoites (pigmented parasites with two or less nuclei), schizonts (pigmented parasites with 
more than two nuclei), or gametocytes as previously described [9].

\section{Outcome measures}

Fever clearance time (FCT) was defined as that from admission to when the axillary temperature reached, and remained below, $37.5^{\circ} \mathrm{C}$ for more than $48 \mathrm{hrs}$. The time to clearance of $50 \%$ and $90 \%$ of the admission parasitaemia $\left(\mathrm{PCT}_{50}\right.$ and $\mathrm{PCT}_{90}$ respectively) were determined using least squares linear regression of log-transformed data. The time to a parasitaemia $<5$ parasites/ $\mu \mathrm{L}$ and the parasite reduction ratio at 24 hours were also calculated. Early treatment failure was defined as a history of fever on day 3, or the development of severe malaria on day 1 to 3 , parasitaemia day 2 greater than on day 0 , pyrexia $\geq 37.5^{\circ} \mathrm{C}$ day 3 , parasitaemia on day $3 \geq 25 \%$ of day 0 . Late treatment failure was defined as severe malaria after day 3 with associated parasitaemia or pyrexia $\geq 37.5{ }^{\circ} \mathrm{C}$ and parasitaemia present day 4 to day 28. These definitions are derived from the WHO criteria for low to moderate transmission areas [11]. Parasite DNA was extracted from the blood spots collected on filter papers daily and at follow-up and was analysed by nested PCR assays to determine the time to negative PCR and to assess for parasite recrudescences and re-infections $[1,12]$.

\section{Statistical analysis}

Data were analysed using SPSS software, version 14 (SPSS). Normally distributed variables were expressed as the mean and standard deviations and comparisons made using the Student $t$-test. Non-normally distributed variables were expressed as the median and interquartile ranges, and comparisons were made using the MannWhitney U test. Proportions were compared with the Fisher's Exact test. A P value $<0.05$ was considered significant in all cases.

\section{Results}

During the study period, 145 out of 187 patients admitted with malaria had single $P$. knowlesi or $P$. vivax malaria infections confirmed by microscopy and PCR. Of these, 111 (77\%) patients had P. knowlesi and 34 (23\%) had $P$. vivax. There were 82 patients with knowlesi malaria $(73.9 \%)$ who were eligible for, and agreed to have, 28-day WHO follow-up and/or six-hourly sampling. Of these, 49 were enrolled for day 28 follow-up only, 24 for both day 28 follow-up and six-hourly sampling, and nine for six-hourly sampling only. All 33 patients (40\%) who consented to six-hourly sampling completed the study but, of the 73 scheduled for 28-day in vivo assessment, 13 did not attend for at least one scheduled review and were excluded. In the vivax group, $29(85 \%)$ of the 34 patients admitted met study criteria and 23 (79\%) had six- hourly blood films taken during the first 24 hours. The majority of the vivax patients (65\%) were logging-camp workers returning from AsiaPacific countries with a higher incidence of malaria than the Kapit region. Because 14 (61\%) patients left the study area to resume employment elsewhere, there were insufficient numbers for analysis of the WHO 28 day extended in vivo drug response.

Subject characteristics at study entry are summarized in Table 1. The patients in the vivax group were younger, all male and more likely to have had malaria previously. Of the patients in the knowlesi group, most were of Iban ethnicity and had been born in the Kapit division. All of the communities along the large rivers and tributaries were represented.

\section{Clinical and parasitological response}

All patients in the knowlesi group responded to treatment. By symptom questionnaire, 96\% reported symptomatic improvement at 24 hours. Late treatment failure was seen in one patient with vivax malaria with the presence of a rising parasitaemia on day 4 of treatment. During treatment, the maximum recorded temperature was higher in the knowlesi group than the vivax group $\left(38.9^{\circ} \mathrm{C}\right.$ versus $38.4^{\circ} \mathrm{C}$ respectively, $\left.P=0.05\right)$, however the FCTs were similar.

Twenty six out of 33 (79\%) patients in the knowlesi group and 20 of the $23(87 \%)$ patients in the vivax group had a parasitaemia on admission that was $>1,000$ parasite $/ \mu \mathrm{L}$. Assessment of parasite clearance kinetics

Table 1 Demographic features of patients at study entry

\begin{tabular}{|c|c|c|c|}
\hline Variable & $\begin{array}{c}\text { P. knowlesi } \\
(\mathrm{n}=73)\end{array}$ & $\begin{array}{l}\text { P. vivax } \\
(\mathrm{n}=23)\end{array}$ & $P$-value \\
\hline Age (years) & $46.4 \pm 13.42$ & $38.5 \pm 7.62$ & $<0.01$ \\
\hline Male & 58 & 100 & $<0.01$ \\
\hline Iban Ethnicity & 96 & 83 & \\
\hline Self-reported previous malaria* & 22 & 65 & 0.01 \\
\hline Duration of illness (days)* & $5[3-7]$ & $3[1-5]$ & 0.01 \\
\hline Self administered paracetamol & 95 & 78 & 0.03 \\
\hline Taken traditional remedies & 39 & 13 & 0.17 \\
\hline Height (metres) & $1.54 \pm 0.09$ & $1.59 \pm 0.07$ & 0.08 \\
\hline Weight (kilograms) Day 0 & $56.2 \pm 10.4$ & $65.3 \pm 6.89$ & $<0.01$ \\
\hline
\end{tabular}

Data are percentage of patients, mean \pm SD or median [interquartile range] 
was restricted to these patients since parasites had usually cleared before the 6 hour blood sample in those with a baseline parasite density $<1,000 / \mu \mathrm{L}$. The admission geometric mean parasitaemia of this subgroup was 8,527 parasite/ $\mu \mathrm{L}$ and 5,724 parasite/ $\mu \mathrm{L}$ for the knowlesi and vivax patients, respectively.

A mixture of early and late trophozoites (mean $49.5 \%$ and $41.5 \%$ respectively) were observed on the admission blood film in the knowlesi group. However, synchronicity was observed in 19 of the 26 patients with up to $100 \%$ early trophozoites and $88 \%$ late trophozoites, while the proportion of schizonts ranged from 0 to $39 \%$. The changing proportion of stages with time is shown in Figure 1.

The $\mathrm{PCT}_{50}$ and $\mathrm{PCT}_{90}$ were 3.1 and 10.3 hours, respectively, in the knowlesi group (Table 2). Nine (27\%) patients exhibited a rise in parasitaemia between baseline and 6 hours, but there was subsequent rapid clearance in these patients. The median calculated time to a parasite density below routine detection $(<5 / \mu \mathrm{L})$, was 35.8 hours and 62.1 hours in the knowlesi and vivax groups, respectively (see Figure 2 ).

The parasite reduction ratio at 24 hours was significantly greater in the knowlesi group than the vivax group (99.4 [97.0-99.9]\% vs 95.9 [88.6-98.4]\%, $P<0.01$ ). The percentages of patients with a negative parasitaemia at 24 hours were 33 (95\% CI: 16-31) and 4.6 (95\% CI: $0-14$ ) for knowlesi and vivax, respectively.

To explore $P$. knowlesi dynamics, synchronous infections were selected, as defined by early or late trophozoite stages comprising $>75 \%$ of all parasite forms (knowlesi group $\mathrm{n}=13$ and 6 respectively; vivax group $\mathrm{n}=6$ and 1 ). There was no significant differences between early and late trophozoite predominant parasitaemias for the $\mathrm{PCT}_{50}$ and $\mathrm{PCT}_{90}$ in the knowlesi group [early trophozoites > 75\% group: $\mathrm{PCT}_{50} 2.1$ (95\% CI:

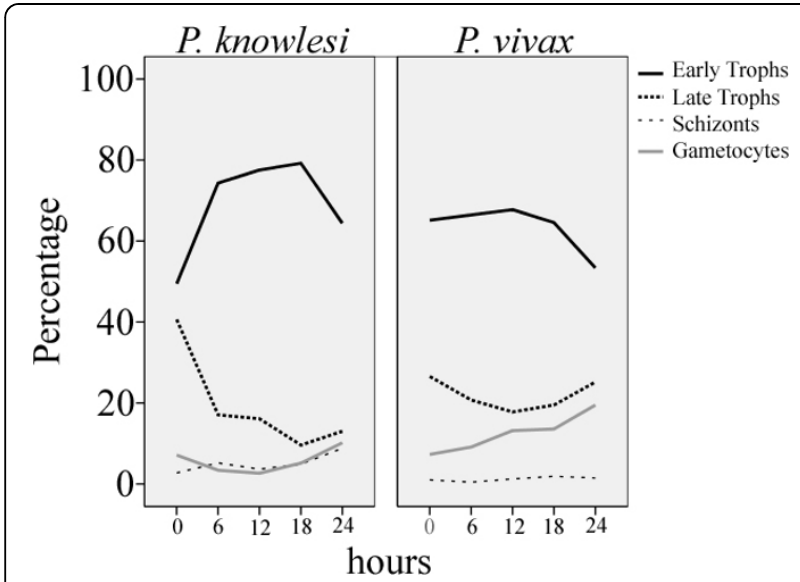

Figure 1 Change in mean parasite stage distribution during the first $\mathbf{2 4}$ hours of treatment with oral chloroquine.
1.8-2.5) hours; $\mathrm{PCT}_{90} 7.0$ (95\% CI: 6.1-8.28) hours. Late trophozoites $>75 \%$ group: $\mathrm{PCT}_{50} 2.2$ (95\% CI: 1.7-3.3) hours; $\mathrm{PCT}_{90} 7.3$ (95\% CI: 5.5-11.0) hours].

Three hourly slides performed in nine selected patients to determine stage specific clearance times found the $\mathrm{PCT}_{50}$ and $\mathrm{PCT}_{90}$ for late trophozoites to be shorter $\left(\mathrm{PCT}_{50} 2.5\right.$ [95\% CI: 2.3-2.9]) than other asexual stages, while gametocytes took longer to clear $\left(\mathrm{PCT}_{50}\right.$ 10.4 [95\% CI: 9.0 - 12.2] hours; $\mathrm{PCT}_{90} 34.4$ [95\% CI: 29.9-40.4]).

The calculated parasitaemia at the time of fever clearance was significantly lower in the knowlesi group $(P<0.01)$. The fever and parasites cleared at a similar time after treatment in knowlesi infections, while the fever cleared sooner than parasites in the vivax group.

\section{In vivo response}

Parasite clearance, detected by nested PCR assays, took a median of 3 [interquartile range: 2-3] days in the knowlesi group. All knowlesi patients were negative for malaria by PCR on days 7, 14, 21 and 28 indicating that there was no evidence of resistance of P. knowlesi to chloroquine and primaquine, re-infection or recrudescence.

\section{Discussion}

The present data demonstrate that conventional doses of chloroquine are associated with initial parasite clearance that is both faster in knowlesi malaria than vivax malaria and amongst the most rapid reported for any human malaria [13-17]. No recurrences of knowlesi parasitaemia were detected during the 28-day follow-up period, including by PCR, suggesting either a low intensity of local transmission of $P$. knowlesi or prophylactic efficacy of relatively low plasma chloroquine concentrations in the tail of the terminal elimination phase. One early treatment failure occurred in the vivax group which was likely due to the increased incidence of chloroquine-resistant $P$. vivax in Java, Indonesia, where the patient had resided until three months prior to hospital admission [18]. The exclusion of $\mathrm{R} 1$ resistance in the vivax group was not possible since too few patients completed follow up.

Although approximately one-third of the knowlesi patients experienced a transient rise in parasitaemia during the first 6 hours compared with only 13\% of patients from the vivax group, this is likely to reflect several factors. First, the divided dose chloroquine regimen produces peak plasma concentrations that occur well after the first dose. Secondly, even if adequate plasma concentrations were reached quickly, the stage specificity of chloroquine [19] would allow late stage parasites to mature to schizogony during at least the first few hours of treatment with production of early ring forms that, as 
Table 2 Data relating to post-treatment clinical and parasitological response

\begin{tabular}{|c|c|c|c|}
\hline Variable & P. knowlesi $(\mathrm{n}=33)$ & P. vivax $(\mathrm{n}=23)$ & $P$ value \\
\hline Admission parasite count (geometric mean per microlitre) & $3724(1,845-7480)$ & $4650(2,752-7785)$ & 0.6 \\
\hline Fever clearance time (hours) & $26.5[16-34]$ & $20.8[12-32]$ & 0.32 \\
\hline Percentage given paracetamol during hospital stay & 79 & 52 & 0.05 \\
\hline Hours before fever clearance & $6[2-13]$ & $4[1-12]$ & 0.48 \\
\hline Time to clear $50 \%$ of admission parasite count (hours) & $3.1(2.84-3.43)$ & $6.3(5.3-7.8)$ & 0.02 \\
\hline Time to clear $90 \%$ of admission parasite count (hours) & $10.3(9.4-11.4)$ & $20.9(17.6-25.9)$ & 0.02 \\
\hline Calculated time to parasite count $<5$ per microlitre (hours) & $35.8[30.8-51.2]$ & $62.1[43.0-80.2]$ & $<0.01$ \\
\hline Parasite reduction ratio at 24 hours & $99.3[50-100]$ & $95.9[58-100]$ & $<0.01$ \\
\hline Percentage negative at 24 hours & $33(16-31)$ & $4.6(0-14)$ & \\
\hline \multicolumn{4}{|l|}{ Parasite stages $(\%$, range $)$} \\
\hline Immature trophozoites & $49.5(3.1-100)$ & $64.4(2.7-98.9)$ & 0.06 \\
\hline Pigmented trophozoites & $41.5(0-88.4)$ & $28.4(19.1-37.6)$ & 0.09 \\
\hline Schizonts & $4.0(0-38.6)$ & $1.9(0-36.3)$ & $<0.01$ \\
\hline Gametocytes & $5.6(0-32.8)$ & $7.1(0-53.7)$ & 0.15 \\
\hline \multicolumn{4}{|l|}{ Slope of curve for natural logarithm parasite count } \\
\hline All & $0.223 \pm 0.02$ & $0.110 \pm 0.02$ & 0.02 \\
\hline Immature trophozoites & $0.182 \pm 0.02$ & $0.127 \pm 0.03$ & NT \\
\hline Mature trophozoites & $0.269 \pm 0.02$ & $0.147 \pm 0.03$ & NT \\
\hline Schizont & $0.191 \pm 0.02$ & $0.02 \pm 0.02$ & NT \\
\hline Gametocyte & $0.061 \pm 0.01$ & $0.012 \pm 0.03$ & NT \\
\hline
\end{tabular}

Data are mean (range), mean \pm SD or median [interquartile range] unless stated otherwise. NT not tested.

evidenced by previous own stage specific clearance times, are also relatively resistant. Thirdly, the 24 hour lifecycle of $P$. knowlesi is half that of $P$. vivax which means that, consistent with the relatively high parasite densities that are regularly observed in P. knowlesi infections [9], the more rapid maturation of late stage parasites would increase the likelihood of an initial rise in parasitaemia amongst the knowlesi patients. Indeed, early studies with chloroquine indicated that $P$. malariae, with an erythrocytic replication cycle of 72 hours, cleared more slowly than P. vivax [20]. Lastly, since most of the knowlesi patients denied a previous history of malaria, a lack of immunity might also have attenuated the effect of chloroquine on initial parasite clearance.

The study participants were aged $>15$ years and lived and/or worked on tributaries of the Rejang river in close proximity to the jungle where long-tailed and pig-tailed macaques have been sighted. Despite this, previous malaria infections, and in particular recent infections, were unusual (reported by only 16 of 73 or $22 \%$ of the knowlesi malaria patients). This suggests a low level of 


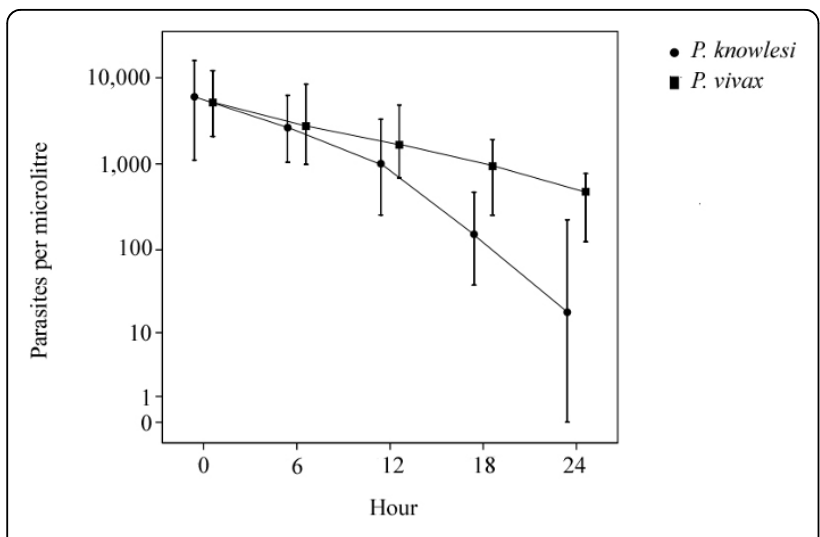

Figure 2 Parasite clearance during the first 24 hours of treatment with oral chloroquine. Graph shows median and 25\%$75 \%$ interquartile ranges.

knowlesi malaria transmission and, therefore, low background levels of immunity in the population. The successful response to treatment with chloroquine and primaquine also implies that these infections are chloroquine and primaquine naïve, supporting a zoonotic mode of transmission.

FCTs and PCTs were similar in the knowlesi group, while fever cleared before parasitaemia in the vivax group. This suggests that knowlesi malaria has a higher pyrogenic density than vivax malaria but it may also reflect differences in immune status between the two groups. Under Malaysian Ministry of Health treatment guidelines, oral primaquine was administered to knowlesi malaria patients for two days in this study as a gametocidal drug. The effect of this on both asexual and sexual forms of $P$. knowlesi is difficult to quantify. Using primaquine would seem unnecessary since gametocytes appeared sensitive to chloroquine in the first 24 hours. $P$. knowlesi is not known to have a hypnozoite stage [21]. If persistent liver stages did occur, and consistent with use of primaquine to prevent relapses of vivax and ovale malaria, longer duration treatment would be probably be necessary. Furthermore, there have been three case reports of chloroquine alone being successful in the treatment of knowlesi malaria [6,22,23].

Besides chloroquine, other anti-malarial agents have been used with apparent success for knowlesi malaria. These include mefloquine [24], quinine [10,25], atovaquone/proguanil [26] and sulphadoxine-pyrimethamine [1]. In animal models and in vitro drug studies, P. knowlesi is sensitive to most antimalarial drugs including clindamycin [27], naphthoquine [28], sulphonamides [29], tetracyclines [30], trimethoprim [31] and erythromycin [32]. In Rhesus macaques, a high level of innate resistance to mefloquine has been seen with the $P$. knowlesi $\mathrm{W} 1$ strain, while proguanil and pyrimethamine resistance were inducible in experiments with the
P. knowlesi Nuri strain [33-35]. Although knowlesi sensitivity to artemisinin derivatives has not been reported in man, it seems very likely that it would be successful as seen in experiments in Rhesus macaques [28,36,37].

In the 1980 's, oral chloroquine was thought to be more effective than oral quinine as treatment for chloroquine sensitive falciparum malaria [38]. Chloroquine was also effective and well tolerated when administered parentally or via nasogastric tube in severe falciparum malaria [39]. It may be that the use of chloroquine for severe knowlesi malaria is effective, provided that the possibility of chloroquine-resistant $P$. falciparum or $P$. vivax co-infection had been excluded. Further studies using alternative anti-malarial drugs, including artemisinin derivatives, would be desirable to define optimal management strategies for $P$. knowlesi.

\section{Conclusion}

Chloroquine is an inexpensive, highly effective and welltolerated anti-malarial drug for the treatment of naturally-acquired uncomplicated knowlesi malaria in humans. There is currently no evidence of drug-resistant $P$. knowlesi, reflecting a zoonotic mode of transmission and thus an absence of prior drug pressure. Further carefully-designed studies are required to determine whether chloroquine is effective in severe knowlesi malaria.

\section{List of abbreviations}

FCT: fever clearance time; PCT: parasite clearance time; 95\% Cl: 95\% confidence interval; PCR: polymerase chain reaction.

\section{Competing interests}

The authors declare that they have no competing interests.

\section{Authors' contributions}

BS and TMED designed this study and wrote the paper with CD and JC-S. $C D$ and MZR recruited and managed the patients. CD was responsible for collection and entry of clinical and laboratory data, and together with TMED, analysed the data. SKZ and PCSD, supervised by JC-S, conducted molecular detection assays. All authors read and approved the final manuscript.

\section{Acknowledgements}

We would like to thank the nursing staff of the Medical Ward, medical assistants at the Emergency Department and the directors, doctors and laboratory staff of Kapit Hospital and Kapit Polyclinic for their assistance in patient recruitment and collection of samples, Roynston Albert Julin and Bruce Kini Jesse for their assistance in processing and transfer of samples to Kuching and the patients who participated in this study. TMED is supported by a National Health and Medical Research Foundation of Australia Practitioner Fellowship. This project was supported by a grant from the Wellcome Trust (no 078538/Z/05/Z).

\section{Author details}

${ }^{1}$ Malaria Research Centre, Faculty of Medicine \& Health Sciences, Universiti Malaysia Sarawak, 93150 Kuching, Sarawak, Malaysia. ${ }^{2}$ University of Western Australia, Department of Medicine, Fremantle Hospital, PO Box 480, Fremantle, WA 6959, Australia. ${ }^{3}$ Kapit Hospital, 96800 Kapit, Sarawak, Malaysia.

Received: 11 May 2010 Accepted: 19 August 2010 Published: 19 August 2010 


\section{References}

1. Singh B, Kim Sung L, Matusop A, Radhakrishnan A, Shamsul SS, Cox-Singh J, Thomas A, Conway DJ: A large focus of naturally acquired Plasmodium knowlesi infections in human beings. Lancet 2004, 363:1017-1024.

2. Cox-Singh J, Davis TM, Lee KS, Shamsul SS, Matusop A, Ratnam S, Rahman HA, Conway DJ, Singh B: Plasmodium knowlesi malaria in humans is widely distributed and potentially life threatening. Clin Infect Dis 2008, 46:165-171.

3. Cox-Singh J, Singh B: Knowlesi malaria: newly emergent and of public health importance? Trends Parasitol 2008, 24:406-410.

4. Putaporntip C, Hongsrimuang T, Seethamchai S, Kobasa T, Limkittikul K, Cui $L$, Jongwutiwes $S$ : Differential prevalence of Plasmodium infections and cryptic Plasmodium knowlesi malaria in humans in Thailand. J Infect Dis 2009, 199:1143-1150.

5. Luchavez J, Espino F, Curameng P, Espina R, Bell D, Chiodini P, Nolder D, Sutherland C, Lee KS, Singh B: Human Infections with Plasmodium knowlesi, the Philippines. Emerg Infect Dis 2008, 14:811-813.

6. Jongwutiwes S, Putaporntip C, Iwasaki T, Sata T, Kanbara H: Naturally acquired Plasmodium knowlesi malaria in human, Thailand. Emerg Infect Dis 2004, 10:2211-2213.

7. Tan CH, Vythilingam I, Matusop A, Chan ST, Singh B: Bionomics of Anopheles latens in Kapit, Sarawak, Malaysian Borneo in relation to the transmission of zoonotic simian malaria parasite Plasmodium knowlesi. Malar J 2008, 7:52

8. Vythilingam I, Tan CH, Asmad M, Chan ST, Lee KS, Singh B: Natural transmission of Plasmodium knowlesi to humans by Anopheles latens in Sarawak, Malaysia. Trans R Soc Trop Med Hyg 2006, 100:1087-1088.

9. Lee KS, Cox-Singh J, Singh B: Morphological features and differential counts of Plasmodium knowlesi parasites in naturally acquired human infections. Malar J 2009, 8:73

10. Daneshvar C, Davis TM, Cox-Singh J, Rafa'ee MZ, Zakaria SK, Divis PC, Singh B: Clinical and laboratory features of human Plasmodium knowlesi infection. Clin Infect Dis 2009, 49:852-860.

11. WHO: Monitoring Antimalarial Drug Resistance. 2001, WHO/CDS/CSR/EPH/ 200217

12. Cox-Singh J, Mahayet S, Abdullah MS, Singh B: Increased sensitivity of malaria detection by nested polymerase chain reaction using simple sampling and DNA extraction. Int J Parasitol 1997, 27:1575-1577.

13. Pukrittayakamee $S$, Chantra A, Simpson JA, Vanijanonta S, Clemens R, Looareesuwan S, White NJ: Therapeutic responses to different antimalarial drugs in vivax malaria. Antimicrob Agents Chemother 2000, 44:1680-1685.

14. Mayxay M, Khanthavong M, Lindegardh N, Keola S, Barends M, Pongvongsa T, Yapom R, Annerberg A, Phompida S, Phetsouvanh R, White NJ, Newton PN: Randomized comparison of chloroquine plus sulfadoxine-pyrimethamine versus artesunate plus mefloquine versus artemether-lumefantrine in the treatment of uncomplicated falciparum malaria in the Lao People's Democratic Republic. Clin Infect Dis 2004, 39:1139-1147.

15. Dunne MW, Singh N, Shukla M, Valecha N, Bhattacharyya PC, Dev V, Patel K, Mohapatra MK, Lakhani J, Benner R, Lele C, Patki K: A multicenter study of azithromycin, alone and in combination with chloroquine, for the treatment of acute uncomplicated Plasmodium falciparum malaria in India. J Infect Dis 2005, 191:1582-1588.

16. Pukrittayakamee S, Imwong M, Looareesuwan S, White NJ: Therapeutic responses to antimalarial and antibacterial drugs in vivax malaria. Acta Trop 2004, 89:351-356

17. Phan GT, de Vries PJ, Tran BQ, Le HQ, Nguyen NV, Nguyen TV, Heisterkamp SH, Kager PA: Artemisinin or chloroquine for blood stage Plasmodium vivax malaria in Vietnam. Trop Med Int Health 2002, 7:858-864.

18. Baird JK, Basri H, Subianto B, Fryauff DJ, McElroy PD, Leksana B, Richie TL, Masbar S, Wignall FS, Hoffman SL: Treatment of chloroquine-resistant Plasmodium vivax with chloroquine and primaquine or halofantrine. $J$ Infect Dis 1995, 171:1678-1682.

19. ter Kuile F, White NJ, Holloway P, Pasvol G, Krishna S: Plasmodium falciparum: in vitro studies of the pharmacodynamic properties of drugs used for the treatment of severe malaria. Exp Parasitol 1993, 76:85-95.

20. Young MD, Eyles DE: The efficacy of chloroquine, quinacrine, quinine and totaquine in the treatment of Plasmodium malariae infections (quartan malaria). Am J Trop Med 1948, s1-28:23-28.
21. Krotoski WA, Collins WE: Failure to detect hypnozoites in hepatic tissue containing exoerythrocytic schizonts of Plasmodium knowlesi. Am J Trop Med Hyg 1982, 31:854-856.

22. van Hellemond JJ, Rutten M, Koelewijn R, Zeeman AM, Verweij JJ, Wismans PJ, Kocken CH, van Genderen PJ: Human Plasmodium knowlesi infection detected by rapid diagnostic tests for malaria. Emerg Infect Dis 2009, 15:1478-1480.

23. Ng OT, Ooi EE, Lee CC, Lee PJ, Ng LC, Pei SW, Tu TM, Loh JP, Leo YS: Naturally acquired human Plasmodium knowlesi infection, Singapore. Emerg Infect Dis 2008, 14:814-816.

24. Bronner U, Divis PC, Farnert A, Singh B: Swedish traveller with Plasmodium knowlesi malaria after visiting Malaysian Borneo. Malar J 2009, 8:15.

25. Kantele A, Marti H, Felger I, Muller D, Jokiranta TS: Monkey malaria in a European traveler returning from Malaysia. Emerg Infect Dis 2008, 14:1434-1436.

26. Figtree $M$, Lee R, Bain L, Kennedy T, Mackertich S, Urban M, Cheng Q, Hudson BJ: Plasmodium knowlesi in Human, Indonesian Borneo. Emerg Infect Dis 16:672-674.

27. Powers KG, Aikawa M, Nugent KM: Plasmodium knowlesi: morphology and course of infection in rhesus monkeys treated with clindamycin and its N-demethyl-4'-pentyl analog. Exp Parasitol 1976, 40:13-24

28. Wang JY, Ding DB, Li GF, Zhao JH: [Therapeutic efficacy of naphthoquine phosphate combined with artemisinine against Plasmodium knowlesi] (in Chinese). Zhongguo Ji Sheng Chong Xue Yu Ji Sheng Chong Bing Za Zhi 2008, 26:442-444.

29. Ray AP, Nair CP: Studies on Nuri Strain of $P$. knowlesi. IX. Susceptibility to sulphonamide substituted dihydrotriazine, sulphadiazine and mepacrine. Indian J Malariol 1955, 9:197-202.

30. Dutta GP, Singh PP: Blood schizontocidal activity of some antibiotics against Plasmodium knowlesi infection in Assamese monkey. Indian J Med Res 1979, 70(Suppl):91-94.

31. Rothe WE, Jacobus DP, Walter WG: Treatment of trophozoite-induced Plasmodium knowlesi infection in the rhesus monkey with trimethoprim and sulfalene. Am J Trop Med Hyg 1969, 18:491-494.

32. Warhurst DC, Robinson BL, Peters W: The blood schizontocidal action of erythromycin against Plasmodium knowlesi infections in Macaca mulatta. Ann Trop Med Parasitol 1983, 77:231-237.

33. Tripathi R, Awasthi A, Dutta GP: Mefloquine resistance reversal action of ketoconazole - a cytochrome P450 inhibitor, against mefloquineresistant malaria. Parasitology 2005, 130:475-479.

34. Singh J, Ray AP, Basu PC, Nair CP: Acquired resistance to proguanil in Plasmodium knowlesi. Trans R Soc Trop Med Hyg 1952, 46:639-649.

35. Singh J, Nair CP, Ray AP: Studies on Nuri strain of $P$. knowlesi. V. Acquired resistance to pyrimethamine. Indian J Malariol 1954, 8:187-195.

36. Shi YL, Li GF, Zhao JH, Yang JD, Ding DB: Schizontocidal effects of oral artesunate on Plasmodium berghei in mice and $P$ knowlesi in monkeys. Zhongguo Yao Li Xue Bao 1999, 20:755-758.

37. Bajpai R, Dutta GP, Vishwakarma RA: Blood schizontocidal activity of a new antimalarial drug, arteether (alpha/beta), against Plasmodium knowlesi in rhesus monkeys. Trans R Soc Trop Med Hyg 1989, 83:484.

38. Salako LA, Sowunmi A, Laoye OJ: Evaluation of the sensitivity in vivo and in vitro of Plasmodium falciparum malaria to quinine in an area of full sensitivity to chloroquine. Trans R Soc Trop Med Hyg 1988, 82:366-368.

39. White NJ, Miller KD, Churchill FC, Berry C, Brown J, Williams SB, Greenwood BM: Chloroquine treatment of severe malaria in children. Pharmacokinetics, toxicity, and new dosage recommendations. N Engl Med 1988, 319:1493-1500.

doi:10.1186/1475-2875-9-238

Cite this article as: Daneshvar et al: Clinical and parasitological response to oral chloroquine and primaquine in uncomplicated human Plasmodium knowlesi infections. Malaria Journal 2010 9:238. 